\title{
Open-flavor strong decays of open-charm and open-bottom mesons in the ${ }^{3} P_{0}$ model
}

\author{
J. Ferretti ${ }^{1,2}$ and E. Santopinto ${ }^{3, *}$ \\ ${ }^{1}$ Institute of Theoretical Physics, Chinese Academy of Sciences, Beijing 100190, China \\ ${ }^{2}$ Dipartimento di Fisica and INFN, "Sapienza" Università di Roma, \\ Piazzale A. Moro 2, 00185 Roma, Italy \\ ${ }^{3}$ INFN, Sezione di Genova, via Dodecaneso 33, 16146 Genova, Italy
}

(Received 31 January 2018; published 20 June 2018)

\begin{abstract}
We provide results for the open-flavor strong decays of open-charm ( $D$ and $\left.D_{\mathrm{s}}\right)$ and open-bottom $\left(B, B_{\mathrm{s}}\right.$, and $\left.B_{\mathrm{c}}\right)$ mesons. The decays are calculated in a modified version of the ${ }^{3} P_{0}$ pair-creation model, assuming harmonic oscillator wave functions. The spectra of open-charm and open-bottom mesons used in the calculations are computed within Godfrey and Isgur's relativized quark model. Quantum number assignments are also provided. Our results are compared with the existing experimental data.
\end{abstract}

DOI: 10.1103/PhysRevD.97.114020

\section{INTRODUCTION}

Since the discovery of the $J / \Psi$ and $\Upsilon$ resonances in the 1970s, heavy meson physics (including the physics of charmonia [1,2], bottomonia [3,4], open-charm [5,6], and open-bottom [7-9] mesons) has been extensively studied and still is the subject of intensive theoretical and experimental research [10,11]. Recently, both the charmonium and bottomonium spectra have been enriched by the discovery of new particles $[10,11]$; also, the knowledge of open-charm and open-bottom mesons has improved substantially with the experimental observation of new resonances, including the $D_{0}^{*}(2400)[12,13], D_{1}(2430)^{0}$ [13], and $B_{1}(5721)$ [14,15]. See Table I. The properties and quantum numbers of a large part of the newly observed open-charm and open-bottom mesons are still not well established. Some examples are $D_{J}^{*}(2600)$ [10,16], $D(2740)^{0}[10,17]$, and $B_{J}(5970)^{0}[10,18]$. This has led to remarkable theoretical efforts to provide the experimentalists predictions for the spectrum, decay modes, and so on and also attempts to make quark model assignments for new observed states.

Important information on mesons can be extracted from their possible decay modes, including electromagnetic, weak, and strong decays. The possibility of providing a theoretical description of (open- and hidden-flavor) strong decays relies mainly on phenomenological models, because

\footnotetext{
*santopinto@ge.infn.it
}

Published by the American Physical Society under the terms of the Creative Commons Attribution 4.0 International license. Further distribution of this work must maintain attribution to the author(s) and the published article's title, journal citation, and DOI. Funded by SCOAP. the operators that describe the strong transitions between hadrons, arising from nonperturbative QCD, are essentially unknown. In the open-flavor case, they include, among other things, "hadrodynamic" models, pair-creation models, elementary meson emission models, heavy meson effective theories, and Lagrangian approaches with heavy-quark and chiral symmetries [19-27]. For a review, see Ref. [28].

TABLE I. Experimental total decay widths and masses of $D$, $D_{s}, B$, and $B_{s}$ mesons, extracted from the particle data group (PDG) [10]. There are no data available for $B_{\mathrm{c}}$ states above the $B D$ threshold. States labeled by $\$$ are omitted from the PDG summary table [10].

\begin{tabular}{llcc}
\hline \hline State & \multicolumn{2}{l}{$J^{P}$ Experimental mass $(\mathrm{MeV})$} & $\Gamma_{\text {tot }}^{\text {exp }}(\mathrm{MeV})$ \\
\hline$D^{*}(2007)^{0}$ & $1^{-}$ & $2006.85 \pm 0.05$ & $<2.1$ \\
$D_{0}^{*}(2400)^{0}$ & $0^{+}$ & $2318 \pm 29$ & $267 \pm 40$ \\
$D_{1}(2420)^{0}$ & $1^{+}$ & $2420.8 \pm 0.5$ & $31.7 \pm 2.5$ \\
$D_{1}(2430)^{0} \$$ & $1^{+}$ & $2427 \pm 26 \pm 25$ & $384_{-75}^{+107} \pm 74$ \\
$D_{s 1}(2460)^{ \pm}$ & $1^{+}$ & $2459.5 \pm 0.6$ & $<3.5$ \\
$D_{s 1}(2536)^{ \pm}$ & $1^{+}$ & $2535.10 \pm 0.06$ & $0.92 \pm 0.03 \pm 0.04$ \\
$D_{2}^{*}(2460)^{0}$ & $2^{+}$ & $2460.7 \pm 0.4$ & $47.5 \pm 1.1$ \\
$D_{0}(2550)^{0} \$$ & $0^{-}$ & $2564 \pm 20$ & $135 \pm 17$ \\
$D_{s 2}^{*}(2573)^{ \pm}$ & $2^{+}$ & $2569.1 \pm 0.8$ & $16.9 \pm 0.8$ \\
$D_{s 1}^{*}(2700)^{ \pm}$ & $1^{-}$ & $2708.3_{-3.4}^{+4.0}$ & $120 \pm 11$ \\
$D_{s 1}^{*}(2860)^{ \pm} \$ 1^{-}$ & $2859 \pm 12 \pm 24$ & $159 \pm 23 \pm 77$ \\
$D_{3}(2750) \$$ & $3^{-}$ & $2763.5 \pm 3.4$ & $66 \pm 5$ \\
$D_{s 3}^{*}(2860) \$$ & $3^{-}$ & $2860.5 \pm 2.6 \pm 6.5$ & $53 \pm 7 \pm 7$ \\
$B_{1}(5721)^{0}$ & $2^{+}$ & $5726.0 \pm 1.3$ & $27.5 \pm 3.4$ \\
$B_{2}^{*}(5747)^{0}$ & $2^{+}$ & $5739.5 \pm 0.7$ & $24.2 \pm 1.7$ \\
$B_{s 1}(5830)^{0}$ & $1^{+}$ & $5828.63 \pm 0.27$ & $0.5 \pm 0.3 \pm 0.3$ \\
$B_{s 2}^{*}(5840)^{0}$ & $2^{+}$ & $5839.85 \pm 0.17$ & $1.47 \pm 0.33$ \\
\hline \hline
\end{tabular}


TABLE II. Pair-creation model parameters for $\mathrm{SU}_{\mathrm{f}}(4)$ and $\mathrm{SU}_{\mathrm{f}}(5)$ sectors, from Refs. [43-45].

\begin{tabular}{lcc}
\hline \hline Parameter & Value in $\mathrm{SU}_{\mathrm{f}}(4)$ & Value in $\mathrm{SU}_{\mathrm{f}}(5)$ \\
\hline$\gamma_{0}$ & 0.510 & 0.732 \\
$\alpha$ & $0.500 \mathrm{GeV}$ & $0.500 \mathrm{GeV}$ \\
$r_{\mathrm{q}}$ & $0.335 \mathrm{fm}$ & $0.335 \mathrm{fm}$ \\
$m_{n}$ & $0.330 \mathrm{GeV}$ & $0.330 \mathrm{GeV}$ \\
$m_{s}$ & $0.550 \mathrm{GeV}$ & $0.550 \mathrm{GeV}$ \\
$m_{c}$ & $1.50 \mathrm{GeV}$ & $1.50 \mathrm{GeV}$ \\
$m_{b}$ & $\cdots$ & $4.70 \mathrm{GeV}$ \\
\hline \hline
\end{tabular}

In this paper, we focus on the ${ }^{3} P_{0}$ pair-creation model, in which the decays proceed via the production of $q \bar{q}$ pairs with vacuum quantum numbers, i.e., $J^{P C}=0^{++}$, somewhere in the hadronic medium [29]. An important feature of the ${ }^{3} P_{0}$ model, apart from its simplicity, is that it provides the gross features of several transitions with only one free parameter, the pair-creation strength $\gamma_{0}$, which is a free constant to be fitted to the experimental data. More recent studies have also discussed the possibility of substituting the constant pair-creation vertex of the model with a more refined one [30-35]. Extensively applied to the study of

TABLE III. Open-flavor strong decay widths (in MeV) for $D$ states. Column 3 gives the values of the masses of the decaying mesons: when available, we use the experimental values from the PDG, denoted by the symbol ${ }^{\dagger}[10]$; otherwise, we consider the predictions of the relativized quark model (QM) for mesons [50]. Columns 4-15 show the decay width contributions (in MeV) from various channels, such as $D \pi, D^{*} \pi$, and so on. The values of the ${ }^{3} P_{0}$ model parameters are given in Table II. The symbol-in the table means that a certain decay is forbidden by selection rules or that the decay cannot take place because it is below threshold. The calculated mixing angles are $\theta_{1 \mathrm{P}}=25.7^{\circ}, \theta_{2 \mathrm{P}}=29.4^{\circ}, \theta_{3 \mathrm{P}}=28.1^{\circ}, \theta_{1 \mathrm{D}}=38.2^{\circ}, \theta_{2 \mathrm{D}}=37.4^{\circ}$, and $\theta_{3 \mathrm{D}}=36.9^{\circ}$.

\begin{tabular}{|c|c|c|c|c|c|c|c|c|c|c|c|c|c|c|}
\hline State & $J^{P}$ & Mass (MeV) & $D \pi$ & $D^{*} \pi$ & $D \rho$ & $D^{*} \rho$ & $D \eta$ & $D^{*} \eta$ & $D \omega$ & $D^{*} \omega$ & $D_{s} K$ & $D_{s}^{*} K$ & $D_{s} K^{*}$ & $D_{s}^{*} K^{*}$ \\
\hline$D^{*}(2007)$ or $D_{1}\left(1^{3} S_{1}\right)$ & $1^{-}$ & $2038,2007^{\dagger}$ & 0 & $\cdots$ & $\cdots$ & $\cdots$ & $\cdots$ & $\cdots$ & $\cdots$ & $\cdots$ & $\cdots$ & $\cdots$ & $\cdots$ & $\cdots$ \\
\hline$D_{0}^{*}(2400)$ or $D_{0}\left(1^{3} P_{0}\right)$ & $0^{+}$ & $2398,2318^{\dagger}$ & 66 & $\ldots$ & $\ldots$ & $\ldots$ & $\ldots$ & $\ldots$ & $\ldots$ & $\ldots$ & $\ldots$ & $\ldots$ & $\ldots$ & $\ldots$ \\
\hline$D_{1}(2420)$ or $D_{1}\left(1 P_{1}\right)$ & $1^{+}$ & $2456,2421^{\dagger}$ & $\cdots$ & 32 & $\cdots$ & $\cdots$ & $\cdots$ & $\cdots$ & $\cdots$ & $\cdots$ & $\cdots$ & $\cdots$ & $\cdots$ & $\cdots$ \\
\hline$D_{1}(2430)$ or $D_{1}\left(1 P_{1}^{\prime}\right)$ & $1^{+}$ & $2467,2427^{\dagger}$ & $\cdots$ & 37 & $\cdots$ & $\cdots$ & $\cdots$ & $\cdots$ & $\cdots$ & $\cdots$ & $\cdots$ & $\cdots$ & $\cdots$ & $\cdots$ \\
\hline$D_{2}^{*}(2460)$ or $D\left(1^{3} P_{2}\right)$ & $2^{+}$ & $2501,2461^{\dagger}$ & 6 & 2 & $\cdots$ & $\cdots$ & 0 & $\cdots$ & $\cdots$ & $\cdots$ & $\cdots$ & $\cdots$ & $\cdots$ & $\cdots$ \\
\hline$D_{0}(2550)$ or $D\left(2^{1} S_{0}\right)$ & $0^{-}$ & $2582,2564^{\dagger}$ & $\cdots$ & 45 & $\cdots$ & $\cdots$ & $\cdots$ & 0 & $\cdots$ & $\ldots$ & $\ldots$ & $\cdots$ & $\ldots$ & $\cdots$ \\
\hline$D_{1}\left(2^{3} S_{1}\right)$ & $1^{-}$ & 2645 & 18 & 36 & 0 & $\ldots$ & 6 & 5 & $\ldots$ & $\ldots$ & 4 & 1 & $\ldots$ & $\ldots$ \\
\hline$D_{1}\left(1^{3} D_{1}\right)$ & $1^{-}$ & 2816 & 20 & 13 & 13 & 1 & 10 & 5 & 4 & 0 & 6 & 2 & $\cdots$ & $\cdots$ \\
\hline$D_{2}\left(1 D_{2}\right)$ & $2^{-}$ & 2816 & $\cdots$ & 25 & 21 & 6 & $\cdots$ & 7 & 7 & 1 & $\cdots$ & 3 & $\cdots$ & $\cdots$ \\
\hline$D_{3}(2750)$ or $D_{3}\left(1^{3} D_{3}\right)$ & $3^{-}$ & $2833,2764^{\dagger}$ & 8 & 5 & 0 & $\cdots$ & 1 & 0 & 0 & $\cdots$ & 0 & 0 & $\cdots$ & $\cdots$ \\
\hline$D_{2}\left(1 D_{2}^{\prime}\right)$ & $2^{-}$ & 2845 & $\cdots$ & 26 & 23 & 5 & $\cdots$ & 8 & 8 & 1 & $\cdots$ & 4 & $\cdots$ & $\cdots$ \\
\hline$D_{1}\left(2 P_{1}\right)$ & $1^{+}$ & 2924 & $\ldots$ & 26 & 20 & 33 & $\ldots$ & 7 & 7 & 11 & $\ldots$ & 4 & 6 & $\cdots$ \\
\hline$D_{0}\left(2^{3} P_{0}\right)$ & $0^{+}$ & 2931 & 18 & $\cdots$ & $\cdots$ & 38 & 2 & $\cdots$ & $\cdots$ & 12 & 0 & $\cdots$ & $\cdots$ & $\cdots$ \\
\hline$D_{2}\left(2^{3} P_{2}\right)$ & $2^{+}$ & 2957 & 13 & 23 & 22 & 45 & 6 & 7 & 7 & 16 & 4 & 4 & 1 & $\cdots$ \\
\hline$D_{1}\left(2 P_{1}^{\prime}\right)$ & $1^{+}$ & 2961 & $\cdots$ & 21 & 14 & 29 & $\cdots$ & 5 & 5 & 9 & $\cdots$ & 3 & 8 & $\cdots$ \\
\hline$D_{0}\left(3{ }^{1} S_{0}\right)$ & $0^{-}$ & 3067 & $\ldots$ & 1 & 4 & 38 & $\ldots$ & 1 & 1 & 13 & $\ldots$ & 3 & 8 & 8 \\
\hline$D_{1}\left(3^{3} S_{1}\right)$ & $1^{-}$ & 3111 & 3 & 2 & 1 & 31 & 0 & 0 & 0 & 11 & 0 & 1 & 5 & 15 \\
\hline$D_{4}\left(1^{3} F_{4}\right)$ & $4^{+}$ & 3113 & 11 & 8 & 4 & 36 & 2 & 1 & 1 & 12 & 1 & 0 & 0 & 1 \\
\hline$D_{2}\left(1^{3} F_{2}\right)$ & $2^{+}$ & 3132 & 10 & 9 & 11 & 12 & 5 & 3 & 4 & 4 & 2 & 2 & 1 & 0 \\
\hline$D_{2}\left(2 D_{2}\right)$ & $2^{-}$ & 3212 & $\ldots$ & 15 & 15 & 30 & $\cdots$ & 5 & 5 & 10 & $\cdots$ & 3 & 3 & 5 \\
\hline$D_{3}\left(2^{3} D_{3}\right)$ & $3^{-}$ & 3226 & 8 & 14 & 16 & 21 & 4 & 5 & 5 & 7 & 3 & 3 & 2 & 9 \\
\hline$D_{2}\left(2 D_{2}^{\prime}\right)$ & $2^{-}$ & 3248 & $\ldots$ & 14 & 13 & 32 & $\cdots$ & 4 & 4 & 11 & $\cdots$ & 3 & 3 & 4 \\
\hline$D_{1}\left(3 P_{1}\right)$ & $1^{+}$ & 3328 & $\cdots$ & 1 & 1 & 10 & $\cdots$ & 0 & 0 & 3 & $\cdots$ & 1 & 3 & 6 \\
\hline$D_{1}\left(2^{3} D_{1}\right)$ & $1^{-}$ & 3231 & 7 & 2 & 0 & 51 & 1 & 0 & 0 & 17 & 0 & 0 & 1 & 4 \\
\hline$D_{0}\left(3^{3} P_{0}\right)$ & $0^{+}$ & 3343 & 1 & $\cdots$ & $\ldots$ & 13 & 0 & $\ldots$ & $\cdots$ & 4 & 1 & $\ldots$ & $\ldots$ & 11 \\
\hline$D_{2}\left(3^{3} P_{2}\right)$ & $2^{+}$ & 3352 & 2 & 1 & 0 & 13 & 0 & 0 & 0 & 5 & 0 & 1 & 1 & 6 \\
\hline$D_{1}\left(3 P_{1}^{\prime}\right)$ & $1^{+}$ & 3360 & $\ldots$ & 1 & 1 & 7 & $\ldots$ & 0 & 1 & 3 & $\ldots$ & 1 & 2 & 8 \\
\hline$D_{3}\left(1^{3} G_{3}\right)$ & $3^{-}$ & 3398 & 5 & 2 & 7 & 15 & 2 & 2 & 2 & 5 & 1 & 1 & 1 & 1 \\
\hline$D_{0}\left(4^{1} S_{0}\right)$ & $0^{-}$ & 3465 & $\cdots$ & 1 & 4 & 11 & $\cdots$ & 1 & 1 & 4 & $\cdots$ & 1 & 1 & 0 \\
\hline$D_{4}\left(2^{3} F_{4}\right)$ & $4^{+}$ & 3466 & 5 & 8 & 10 & 12 & 2 & 3 & 3 & 4 & 2 & 2 & 2 & 4 \\
\hline$D_{2}\left(2^{3} F_{2}\right)$ & $2^{+}$ & 3490 & 3 & 1 & 0 & 38 & 1 & 0 & 0 & 12 & 0 & 0 & 0 & 5 \\
\hline$D_{2}\left(3 D_{2}\right)$ & $2^{-}$ & 3566 & $\ldots$ & 1 & 1 & 3 & $\ldots$ & 0 & 0 & 1 & $\ldots$ & 1 & 1 & 3 \\
\hline$D_{3}\left(3^{3} D_{3}\right)$ & $3^{-}$ & 3578 & 2 & 1 & 0 & 6 & 0 & 0 & 0 & 2 & 0 & 0 & 1 & 2 \\
\hline$D_{2}\left(3 D_{2}^{\prime}\right)$ & $2^{-}$ & 3600 & $\ldots$ & 1 & 1 & 3 & $\ldots$ & 0 & 0 & 1 & $\ldots$ & 1 & 1 & 3 \\
\hline
\end{tabular}


open-flavor strong decays of light mesons $[30,36,37]$ and baryons [38-40], the ${ }^{3} P_{0}$ pair-creation model has also been used to compute the decays of charmonia [41-44], bottomonia $[45,46]$, open-charm $[35,47,48]$, and open-bottom [49] mesons.

The aim of the present paper is to provide a classification of open-charm and open-bottom mesons in terms of their masses, calculated within Godfrey and Isgur's relativized model [50,51]; open-flavor amplitudes, evaluated within a modified version of the ${ }^{3} P_{0}$ pair-creation model [43-45,52]; and quantum number assignments, carried out by comparing our predictions to the existing data.

As widely shown by previous quark model (QM) calculations, we expect to obtain a good overall description of the properties of these mesons, with the possible exception of states close to meson-meson decay thresholds, like $D_{0}^{*}(2400)$ and $D_{s 0}^{*}(2317)$ [53]. Indeed, it is well known that the quenched approximation may fail for states in the region around the opening of meson-meson decay thresholds, where it is believed that continuum-coupling effects may play an important role $[43,44,54-58]$. A study of these particular states in the context of coupled-channel models will be addressed in a future publication.

\section{FORMALISM}

\section{A. ${ }^{3} \boldsymbol{P}_{\mathbf{0}}$ pair-creation model}

In the ${ }^{3} P_{0}$ pair-creation model, the open-flavor strong decay of a hadron $A$ into hadrons $B$ and $C$ takes place in its rest frame, via the creation of an additional $q \bar{q}$ pair characterized by $J^{P C}=0^{++}$quantum numbers $[29,38,59]$. The decay widths $A \rightarrow B C$ are calculated as $[29,37,38]$

$$
\Gamma_{A \rightarrow B C}=\Phi_{A \rightarrow B C}\left(q_{0}\right) \sum_{\ell}\left|\left\langle B C q_{0} \ell J\left|T^{\dagger}\right| A\right\rangle\right|^{2} .
$$

TABLE IV. As Table III, but for $D_{\mathrm{s}}$ mesons. The calculated mixing angles are $\theta_{1 \mathrm{P}}=37.5^{\circ}, \theta_{2 \mathrm{P}}=30.4^{\circ}, \theta_{3 \mathrm{P}}=27.7^{\circ}, \theta_{1 \mathrm{D}}=38.5^{\circ}$, $\theta_{2 \mathrm{D}}=37.7^{\circ}$, and $\theta_{3 \mathrm{D}}=37.2^{\circ}$. In the $1 P_{1}-1 P_{1}^{\prime}$ and $D_{\mathrm{s} 3}^{*}(2860)$ cases, the values in parentheses are calculated by using the relativized $\mathrm{QM}$ predictions for the decaying meson masses.

\begin{tabular}{|c|c|c|c|c|c|c|c|c|c|c|}
\hline State & $J^{P}$ & Mass $(\mathrm{MeV})$ & $D K$ & $D^{*} K$ & $D K^{*}$ & $D^{*} K^{*}$ & $D_{s} \eta^{\prime}$ & $D_{s}^{*} \eta^{\prime}$ & $D_{s} \phi$ & $D_{s}^{*} \phi$ \\
\hline$D_{\mathrm{s} 1}(2460)$ or $D_{\mathrm{s} 1}\left(1 P_{1}\right)$ & $1^{+}$ & $2549,2460^{\dagger}$ & $\cdots$ & $\cdots(46)$ & $\cdots$ & $\cdots$ & $\cdots$ & $\cdots$ & $\cdots$ & $\cdots$ \\
\hline$D_{\mathrm{s} 1}(2536)$ or $D_{\mathrm{s} 1}\left(1 P_{1}^{\prime}\right)$ & $1^{+}$ & $2556,2535^{\dagger}$ & $\ldots$ & $56(54)$ & $\ldots$ & $\ldots$ & $\ldots$ & $\ldots$ & $\ldots$ & $\cdots$ \\
\hline$D_{\mathrm{s} 2}^{*}(2573)$ or $D_{\mathrm{s} 2}\left(1^{3} P_{2}\right)$ & $2^{+}$ & $2591,2569^{\dagger}$ & 4 & 0 & $\cdots$ & $\cdots$ & $\cdots$ & $\cdots$ & $\cdots$ & $\cdots$ \\
\hline$D_{\mathrm{s} 0}\left(2^{1} S_{0}\right)$ & $0^{-}$ & 2675 & $\cdots$ & 53 & $\cdots$ & $\cdots$ & $\cdots$ & $\cdots$ & $\cdots$ & $\cdots$ \\
\hline$D_{\mathrm{s} 1}^{*}(2700)$ or $D_{\mathrm{s} 1}\left(2^{3} S_{1}\right)$ & $1^{-}$ & $2735,2708^{\dagger}$ & 28 & 42 & $\cdots$ & $\cdots$ & $\cdots$ & $\cdots$ & $\cdots$ & $\cdots$ \\
\hline$D_{\mathrm{s} 1}^{*}(2860)$ or $D_{\mathrm{s} 1}\left(1^{3} D_{1}\right)$ & $1^{-}$ & $2898,2859^{\dagger}$ & 43 & 23 & 13 & $\ldots$ & $\ldots$ & $\ldots$ & $\ldots$ & $\cdots$ \\
\hline$D_{\mathrm{s} 3}^{*}(2860)$ or $D_{\mathrm{s} 3}\left(1^{3} D_{3}\right)$ & $3^{-}$ & $2916,2861^{\dagger}$ & $10(14)$ & $5(8)$ & $0(1)$ & $\cdots(5)$ & $\cdots$ & $\ldots$ & $\ldots$ & $\cdots$ \\
\hline$D_{\mathrm{s} 2}\left(1 D_{2}\right)$ & $2^{-}$ & 2900 & $\ldots$ & 40 & 31 & 1 & $\ldots$ & $\ldots$ & $\ldots$ & $\ldots$ \\
\hline$D_{\mathrm{s} 2}\left(1 D_{2}^{\prime}\right)$ & $2^{-}$ & 2926 & $\cdots$ & 43 & 34 & 1 & $\cdots$ & $\cdots$ & $\cdots$ & $\cdots$ \\
\hline$D_{\mathrm{s} 0}\left(2^{3} P_{0}\right)$ & $0^{+}$ & 3005 & 28 & $\cdots$ & $\cdots$ & 51 & 14 & $\cdots$ & $\cdots$ & $\cdots$ \\
\hline$D_{\mathrm{s} 1}\left(2 P_{1}\right)$ & $1^{+}$ & 3018 & $\cdots$ & 37 & 27 & 44 & $\cdots$ & $\ldots$ & 7 & $\cdots$ \\
\hline$D_{\mathrm{s} 1}\left(2 P_{1}^{\prime}\right)$ & $1^{+}$ & 3038 & $\cdots$ & 31 & 20 & 35 & $\cdots$ & $\cdots$ & 10 & $\cdots$ \\
\hline$D_{\mathrm{s} 2}\left(2^{3} P_{2}\right)$ & $2^{+}$ & 3049 & 19 & 33 & 31 & 64 & 1 & $\cdots$ & 0 & $\cdots$ \\
\hline$D_{\mathrm{s} 0}\left(3^{1} S_{0}\right)$ & $0^{-}$ & 3153 & $\cdots$ & 2 & 3 & 50 & .. & 6 & 8 & 3 \\
\hline$D_{\mathrm{s} 4}\left(1^{3} F_{4}\right)$ & $4^{+}$ & 3190 & 17 & 11 & 5 & 60 & 0 & 0 & 0 & 0 \\
\hline$D_{\mathrm{s} 1}\left(3^{3} S_{1}\right)$ & $1^{-}$ & 3194 & 6 & 3 & 0 & 39 & 2 & 5 & 5 & 11 \\
\hline$D_{\mathrm{s} 2}\left(1^{3} F_{2}\right)$ & $2^{+}$ & 3208 & 21 & 17 & 19 & 18 & 3 & 1 & 1 & 4 \\
\hline$D_{\mathrm{s} 2}\left(2 D_{2}\right)$ & $2^{-}$ & 3298 & $\cdots$ & 24 & 22 & 44 & $\cdots$ & 3 & 3 & 5 \\
\hline$D_{\mathrm{s} 1}\left(2^{3} D_{1}\right)$ & $1^{-}$ & 3306 & 13 & 3 & 1 & 74 & 1 & 2 & 1 & 4 \\
\hline$D_{\mathrm{s} 3}\left(2^{3} D_{3}\right)$ & $3^{-}$ & 3311 & 2 & 20 & 23 & 29 & 2 & 1 & 2 & 10 \\
\hline$D_{\mathrm{s} 2}\left(2 D_{2}^{\prime}\right)$ & $2^{-}$ & 3323 & $\cdots$ & 22 & 21 & 47 & $\cdots$ & 3 & 3 & 5 \\
\hline$D_{\mathrm{s} 0}\left(3^{3} P_{0}\right)$ & $0^{+}$ & 3412 & 3 & & $\ldots$ & 15 & 2 & .. & . & 12 \\
\hline$D_{\mathrm{s} 1}\left(3 P_{1}\right)$ & $1^{+}$ & 3416 & $\ldots$ & 3 & 1 & 10 & $\cdots$ & 3 & 3 & 7 \\
\hline$D_{\mathrm{s} 1}\left(3 P_{1}^{\prime}\right)$ & $1^{+}$ & 3433 & $\cdots$ & 2 & 1 & 7 & $\cdots$ & 3 & 2 & 8 \\
\hline$D_{\mathrm{s} 2}\left(3^{3} P_{2}\right)$ & $2^{+}$ & 3439 & 6 & 3 & 1 & 14 & 1 & 2 & 1 & 6 \\
\hline$D_{\mathrm{s} 3}\left(1^{3} G_{3}\right)$ & $3^{-}$ & 3469 & 12 & 12 & 13 & 27 & 2 & 1 & 1 & 1 \\
\hline$D_{\mathrm{s} 0}\left(4^{1} S_{0}\right)$ & $0^{-}$ & 3544 & $\cdots$ & 1 & 4 & 16 & . & 1 & 1 & 1 \\
\hline$D_{\mathrm{s} 4}\left(2^{3} F_{4}\right)$ & $4^{+}$ & 3544 & 7 & 12 & 15 & 18 & 2 & 1 & 2 & 5 \\
\hline$D_{\mathrm{s} 2}\left(2^{3} F_{2}\right)$ & $2^{+}$ & 3562 & 8 & 3 & 2 & 59 & 0 & 0 & 0 & 6 \\
\hline$D_{\mathrm{s} 2}\left(3 D_{2}\right)$ & $2^{-}$ & 3650 & $\ldots$ & 3 & 2 & 3 & . & 1 & 1 & 3 \\
\hline$D_{\mathrm{s} 3}\left(3^{3} D_{3}\right)$ & $3^{-}$ & 3661 & 5 & 3 & 1 & 7 & 0 & 1 & 0 & 3 \\
\hline$D_{\mathrm{s} 2}\left(3 D_{2}^{\prime}\right)$ & $2^{-}$ & 3672 & $\cdots$ & 3 & 1 & 3 & $\cdots$ & 1 & 1 & 3 \\
\hline
\end{tabular}


The coefficient

$$
\Phi_{A \rightarrow B C}\left(q_{0}\right)=2 \pi q_{0} \frac{E_{b}\left(q_{0}\right) E_{c}\left(q_{0}\right)}{M_{a}},
$$

depending on the relative momentum $q_{0}$ between $B$ and $C$ and the energies of the two decay products, $E_{b, c}=\sqrt{M_{b, c}^{2}+q_{0}^{2}}$, is the phase space factor for the decay. We assume harmonic oscillator wave functions, depending on a single oscillator parameter $\alpha$. The final state is characterized by the relative orbital angular momentum $\ell$ between $B$ and $C$ and a total angular momentum $\vec{J}=\vec{J}_{b}+\vec{J}_{c}+\vec{\ell}$.

Following Refs. [43-45,52,60], we introduce a few changes in the ${ }^{3} P_{0}$ model operator, $T^{\dagger}$. These modifications include the substitution of the pair-creation strength, $\gamma_{0}$, with an effective one $[34,43-45,52,60]$,

$$
\gamma_{0}^{\mathrm{eff}}=\frac{m_{n}}{m_{i}} \gamma_{0}
$$

with $i=n$ (i.e., $u$ or $d$ ), $s, c$, and $b$ (see Table II), to suppress heavy quark pair creation. Something similar was also done in Ref. [35], though the authors used a different form for $\gamma_{0}^{\text {eff }}$. We also introduce a Gaussian quark form factor, with parameter $r_{\mathrm{q}}$, because the pair of created quarks has an effective size $[43-45,52,60]$.

The values of the pair-creation model parameters for the $\mathrm{SU}(4)_{\mathrm{f}}$ and $\mathrm{SU}(5)_{\mathrm{f}}$ sectors, reported in Table II, are extracted from Refs. [43-45]. These are the values that we use in our calculations.

\section{B. Godfrey and Isgur's relativized quark model}

The relativized quark model [50,51] is based on an effective potential, the dynamics of which is governed by a one-gluon exchange interaction at short distances plus a long-range linear confining one.

The Hamiltonian of the model is given by [50]

$H=\sqrt{q^{2}+m_{1}^{2}}+\sqrt{q^{2}+m_{2}^{2}}+V_{\text {conf }}+V_{\text {hyp }}+V_{\text {so }}$,

where $m_{1}$ and $m_{2}$ are the masses of the constituent quark and antiquark; $q$ is their relative momentum (with conjugate coordinate $r$ ); $V_{\text {conf }}, V_{\text {hyp }}$, and $V_{\text {so }}$ the confining, hyperfine, and spin-orbit potentials, respectively.

The confining potential is the sum of three terms [50],

$$
V_{\mathrm{conf}}=-\left(\frac{3}{4} c+\frac{3}{4} b r-\frac{\alpha_{\mathrm{s}}(r)}{r}\right) \vec{F}_{1} \cdot \vec{F}_{2}
$$

with $\left\langle q \bar{q}\left|\vec{F}_{1} \cdot \vec{F}_{2}\right| q \bar{q}\right\rangle=-\frac{4}{3}$. The first term is a constant; the second is a spin-independent linear confining one, with parameter $b$; and the third is a Coulomb-like interaction. The hyperfine interaction has the standard form [50]

$$
\begin{aligned}
V_{\text {hyp }}= & -\frac{\alpha_{\mathrm{s}}(r)}{m_{1} m_{2}}\left[\frac{8 \pi}{3} \vec{S}_{1} \cdot \vec{S}_{2} \delta^{3}(\vec{r})\right. \\
& \left.+\frac{1}{r^{3}}\left(\frac{3 \vec{S}_{1} \cdot \vec{r} \vec{S}_{2} \cdot \vec{r}}{r^{2}}-\vec{S}_{1} \cdot \vec{S}_{2}\right)\right] \vec{F}_{i} \cdot \vec{F}_{j}
\end{aligned}
$$

\begin{tabular}{|c|c|c|c|}
\hline Ratio & ${ }^{3} P_{0}$ & HMET and HQCS & Exp. \\
\hline$\frac{\Gamma_{D_{2}^{*}(2460) \rightarrow D \pi}}{\Gamma_{D_{2}^{*}(2460) \rightarrow D^{*} \pi}}$ & 3.0 & 2.29 [27], 2.27 [24] & $1.5-3.0[16,61-63]$ \\
\hline$\frac{\Gamma_{D(2550)}^{\text {of }}}{\Gamma_{D(2600)}^{\text {of }}}$ & 0.64 & $0.85[23]$ & $\cdots$ \\
\hline$\frac{\Gamma_{D_{s 2}^{*}(2573) \rightarrow D^{*} K}}{\Gamma_{D_{s 2}^{*}(2573) \rightarrow D K}}$ & $\approx 0$ & $0.086[24]$ & $<0.33[10]$ \\
\hline$\frac{\Gamma_{D_{s 1}^{*}(2700) \rightarrow D^{*} K}}{\Gamma_{D_{s 1}^{*}(2700) \rightarrow D K}}$ & 1.5 & $0.91[24]$ & $0.91 \pm 0.13 \pm 0.12[10]$ \\
\hline$\frac{\Gamma_{D_{3}^{*}}^{*}(2860) \rightarrow D^{*} K}{\Gamma_{D_{33}^{*}(2860) \rightarrow D K K}}$ & $0.50(0.57)^{\mathrm{a}}$ & $0.39[24]$ & $1.10 \pm 0.15 \pm 0.19[65]$ \\
\hline$\frac{\Gamma\left(B_{2}^{*}(5747) \rightarrow B^{*} \pi\right)}{\Gamma\left(B_{2}^{*}(5747) \rightarrow B \pi\right)}$ & 0.75 & $0.87[24]$ & $1.0 \pm 0.5 \pm 0.8[10,64]$ \\
\hline$\frac{\Gamma\left(B_{s 2}^{*}(5840) \rightarrow B^{*} K\right)}{\Gamma\left(B_{2}^{*}(5747) \rightarrow B K\right)}$ & $\approx 0$ & $0.07[24]$ & $0.093 \pm 0.013 \pm 0.012[10]$ \\
\hline$\frac{\Gamma\left(B_{3}\left(1^{3} D_{3}\right) \rightarrow B^{*} \pi\right)}{\Gamma\left(B_{3}\left(2^{3} D_{3}\right) \rightarrow B \pi\right)}$ & 1.0 & $0.92[24]$ & $\cdots$ \\
\hline$\frac{\Gamma\left(B_{53}\left(1^{3} D_{3}\right) \rightarrow B^{*} K\right)}{\Gamma\left(B_{33}\left(1^{3} D_{3}\right) \rightarrow B K\right)}$ & 0.94 & $0.815[24]$ & $\cdots$ \\
\hline
\end{tabular}

The spin-orbit potential [50],

TABLE V. Strong decay amplitude ratios. Our ${ }^{3} P_{0}$ model results are compared to those of heavy meson effective theories (HMET) of Refs. [23,27], Lagrangian approaches with heavy quark and chiral symmetries (HQCS) of Refs. [24], and the experimental data [10,16,61-64].

${ }^{\mathrm{a}}$ See the caption of Table IV. 


$$
V_{\mathrm{so}}=V_{\mathrm{so}, \mathrm{cm}}+V_{\mathrm{so}, \mathrm{tp}}
$$

is the sum of two contributions, where

$$
V_{\mathrm{so}, \mathrm{cm}}=-\frac{\alpha_{\mathrm{s}}(r)}{r^{3}}\left(\frac{1}{m_{i}}+\frac{1}{m_{j}}\right)\left(\frac{\vec{S}_{i}}{m_{i}}+\frac{\vec{S}_{j}}{m_{j}}\right) \cdot \vec{L} \vec{F}_{i} \cdot \vec{F}_{j}
$$

is the color-magnetic term and

$$
V_{\mathrm{so}, \mathrm{tp}}=-\frac{1}{2 r} \frac{\partial V_{i j \mathrm{conf}}}{\partial r}\left(\frac{\vec{S}_{i}}{m_{i}^{2}}+\frac{\vec{S}_{j}}{m_{j}^{2}}\right) \cdot \vec{L}
$$

the Thomas-precession one.

In the case of states characterized by a quark and antiquark of unequal mass, charge conjugation is not a good quantum number. Therefore, states with different spins but the same angular momentum, $\left|n^{1} L_{J}\right\rangle$ and $\left|n^{3} L_{J}\right\rangle$, can mix via the spin-orbit interaction. For example, this happens in the case of ${ }^{1} P_{1}$ and ${ }^{3} P_{1}$ states, where we consider the linear combinations

TABLE VI. As Table III, but for $B$ mesons. The calculated mixing angles are $\theta_{1 \mathrm{P}}=30.3^{\circ}, \theta_{2 \mathrm{P}}=32.3^{\circ}, \theta_{3 \mathrm{P}}=31.6^{\circ}, \theta_{1 \mathrm{D}}=39.7^{\circ}$, $\theta_{2 \mathrm{D}}=39.0^{\circ}$, and $\theta_{3 \mathrm{D}}=38.6^{\circ}$. The width denoted by the symbol $\$$ is calculated by using the mass of the decaying meson from the Godfrey-Isgur model. If we use the experimental value of the mass and do not introduce a mixing with $B_{1}\left(1 P_{1}^{\prime}\right)$, we get a $B^{*} \pi$ width of

\begin{tabular}{|c|c|c|c|c|c|c|c|c|c|c|c|c|c|c|}
\hline State & $J^{P}$ & Mass $(\mathrm{MeV})$ & $B \pi$ & $B^{*} \pi$ & $B \rho$ & $B^{*} \rho$ & $B \eta$ & $B^{*} \eta$ & $B \omega$ & $B^{*} \omega$ & $B_{s} K$ & $B_{s}^{*} K$ & $B_{s} K^{*}$ & $B_{s}^{*} K^{*}$ \\
\hline$B_{1}(5721)$ or $B_{1}\left(1 P_{1}\right)$ & $1^{+}$ & $5777,5726^{\dagger}$ & $\cdots$ & $55^{\S}$ & $\cdots$ & $\cdots$ & $\cdots$ & $\cdots$ & $\cdots$ & $\cdots$ & $\cdots$ & $\cdots$ & $\cdots$ & $\cdots$ \\
\hline$B_{2}^{*}(5747)$ or $B_{2}\left(1^{3} P_{2}\right)$ & $2^{+}$ & $5796,5740^{\dagger}$ & 4 & 3 & $\cdots$ & $\cdots$ & $\cdots$ & $\cdots$ & $\cdots$ & $\cdots$ & $\cdots$ & $\cdots$ & $\cdots$ & $\cdots$ \\
\hline$B_{0}\left(1^{3} P_{0}\right)$ & $0^{+}$ & 5756 & 117 & $\cdots$ & $\cdots$ & $\cdots$ & $\cdots$ & $\cdots$ & $\cdots$ & $\cdots$ & $\cdots$ & $\cdots$ & $\cdots$ & $\cdots$ \\
\hline$B_{1}\left(1 P_{1}^{\prime}\right)$ & $1^{+}$ & 5784 & $\cdots$ & 73 & $\cdots$ & $\cdots$ & $\cdots$ & $\cdots$ & $\cdots$ & $\cdots$ & $\cdots$ & $\cdots$ & $\cdots$ & $\cdots$ \\
\hline$B_{0}\left(2^{1} S_{0}\right)$ & $0^{-}$ & 5905 & $\cdots$ & 87 & $\cdots$ & $\cdots$ & $\cdots$ & 4 & $\cdots$ & $\cdots$ & $\cdots$ & $\ldots$ & $\ldots$ & $\cdots$ \\
\hline$B_{1}\left(2^{3} S_{1}\right)$ & $1^{-}$ & 5934 & 30 & 60 & $\ldots$ & $\ldots$ & 7 & 6 & $\ldots$ & $\ldots$ & 3 & 1 & $\ldots$ & $\ldots$ \\
\hline$B_{2}\left(1 D_{2}\right)$ & $2^{-}$ & 6095 & $\cdots$ & 49 & 16 & 1 & $\cdots$ & 15 & 4 & 0 & $\cdots$ & 7 & $\cdots$ & $\cdots$ \\
\hline$B_{3}\left(1^{3} D_{3}\right)$ & $3^{-}$ & 6105 & 16 & 16 & 0 & 1 & 1 & 1 & 0 & $\cdots$ & 0 & 0 & $\cdots$ & $\cdots$ \\
\hline$B_{1}\left(1^{3} D_{1}\right)$ & $1^{-}$ & 6110 & 42 & 23 & 11 & 1 & 21 & 10 & 3 & 0 & 11 & 5 & $\cdots$ & $\cdots$ \\
\hline$B_{2}\left(1 D_{2}^{\prime}\right)$ & $2^{-}$ & 6124 & $\cdots$ & 50 & 17 & 2 & $\ldots$ & 16 & 5 & 0 & $\ldots$ & 8 & $\ldots$ & $\ldots$ \\
\hline$B_{1}\left(2 P_{1}\right)$ & $1^{+}$ & 6197 & $\cdots$ & 46 & 31 & 58 & $\cdots$ & 12 & 10 & 19 & $\cdots$ & 7 & $\cdots$ & $\cdots$ \\
\hline$B_{2}\left(2^{3} P_{2}\right)$ & $2^{+}$ & 6213 & 22 & 35 & 21 & 99 & 9 & 13 & 7 & 35 & 6 & 7 & $\cdots$ & $\ldots$ \\
\hline$B_{0}\left(2^{3} P_{0}\right)$ & $0^{+}$ & 6214 & 35 & $\cdots$ & $\cdots$ & 70 & 3 & $\cdots$ & $\cdots$ & 22 & 0 & $\cdots$ & $\cdots$ & $\cdots$ \\
\hline$B_{1}\left(2 P_{1}^{\prime}\right)$ & $1^{+}$ & 6228 & $\cdots$ & 42 & 29 & 48 & $\cdots$ & 10 & 10 & 15 & $\cdots$ & 5 & $\ldots$ & $\cdots$ \\
\hline$B_{0}\left(3^{1} S_{0}\right)$ & $0^{-}$ & 6334 & $\cdots$ & 11 & 16 & 59 & $\cdots$ & 0 & 6 & 21 & $\cdots$ & 2 & 11 & 6 \\
\hline$B_{1}\left(3^{3} S_{1}\right)$ & $1^{-}$ & 6355 & 6 & 9 & 7 & 53 & 0 & 0 & 3 & 19 & 0 & 1 & 9 & 16 \\
\hline$B_{4}\left(1^{3} F_{4}\right)$ & $4^{+}$ & 6364 & 19 & 20 & 4 & 80 & 3 & 3 & 1 & 26 & 1 & 1 & 0 & 1 \\
\hline$B_{2}\left(1^{3} F_{2}\right)$ & $2^{+}$ & 6387 & 20 & 15 & 23 & 27 & 10 & 7 & 8 & 9 & 5 & 4 & 1 & 0 \\
\hline$B_{2}\left(2 D_{2}\right)$ & $2^{-}$ & 6450 & $\cdots$ & 25 & 24 & 56 & $\cdots$ & 9 & 8 & 19 & $\cdots$ & 5 & 5 & 9 \\
\hline$B_{3}\left(2^{3} D_{3}\right)$ & $3^{-}$ & 6459 & 12 & 18 & 26 & 37 & 6 & 9 & 8 & 12 & 4 & 5 & 2 & 17 \\
\hline$B_{1}\left(2^{3} D_{1}\right)$ & $1^{-}$ & 6475 & 15 & 6 & 0 & 100 & 3 & 1 & 0 & 33 & 0 & 0 & 2 & 7 \\
\hline$B_{2}\left(2 D_{2}^{\prime}\right)$ & $2^{-}$ & 6486 & $\ldots$ & 25 & 22 & 60 & .. & 8 & 7 & 20 & . & 4 & 6 & 8 \\
\hline$B_{1}\left(3 P_{1}\right)$ & $1^{+}$ & 6557 & $\ldots$ & 7 & 3 & 9 & $\cdots$ & 0 & 1 & 3 & . & 1 & 5 & 11 \\
\hline$B_{2}\left(3^{3} P_{2}\right)$ & $2^{+}$ & 6570 & 6 & 7 & 0 & 16 & 1 & 0 & 0 & 6 & 0 & 0 & 3 & 10 \\
\hline$B_{1}\left(3 P_{1}^{\prime}\right)$ & $1^{+}$ & 6585 & .. & 7 & 3 & 5 & .. & 0 & 1 & 2 & .. & 1 & 4 & 12 \\
\hline$B_{0}\left(3^{3} P_{0}\right)$ & $0^{+}$ & 6590 & 6 & $\cdots$ & $\cdots$ & 5 & 0 & $\cdots$ & $\cdots$ & 2 & 0 & $\cdots$ & . & 18 \\
\hline$B_{3}\left(1^{3} G_{3}\right)$ & $3^{-}$ & 6622 & 10 & 9 & 15 & 38 & 5 & 4 & 5 & 12 & 2 & 2 & 2 & 2 \\
\hline$B_{4}\left(2^{3} F_{4}\right)$ & $4^{+}$ & 6679 & 5 & 9 & 17 & 22 & 3 & 5 & 6 & 7 & 2 & 3 & 2 & 7 \\
\hline$B_{0}\left(4^{1} S_{0}\right)$ & $0^{-}$ & 6687 & $\cdots$ & 0 & 6 & 16 & $\cdots$ & 1 & 2 & 5 & . & 1 & 2 & 2 \\
\hline$B_{2}\left(2^{3} F_{2}\right)$ & $2^{+}$ & 6704 & 9 & 5 & 12 & 69 & 2 & 1 & 0 & 23 & 0 & 0 & 0 & 9 \\
\hline$B_{2}\left(3 D_{2}\right)$ & $2^{-}$ & 6767 & $\cdots$ & 6 & 1 & 2 & - & 1 & 0 & 1 & - & 0 & 1 & 4 \\
\hline$B_{3}\left(3^{3} D_{3}\right)$ & $3^{-}$ & 6775 & 5 & 5 & 1 & 6 & 1 & 1 & 0 & 2 & 0 & 0 & 1 & 4 \\
\hline$B_{2}\left(3 D_{2}^{\prime}\right)$ & $2^{-}$ & 6800 & . & 5 & 1 & 2 & . & 1 & 0 & 1 & . & 0 & 1 & 4 \\
\hline$B_{3}\left(2^{3} G_{3}\right)$ & $3^{-}$ & 6909 & 5 & 4 & 2 & 38 & 1 & 1 & 0 & 13 & 0 & 0 & 0 & 7 \\
\hline$B_{0}\left(4^{3} P_{0}\right)$ & $0^{+}$ & 6954 & 1 & $\ldots$ & $\cdots$ & 3 & 0 & $\ldots$ & $\ldots$ & 1 & 0 & $\ldots$ & $\cdots$ & 3 \\
\hline$B_{4}\left(3^{3} F_{4}\right)$ & $4^{+}$ & 6966 & 3 & 4 & 1 & 3 & 1 & 1 & 0 & 1 & 0 & 0 & 0 & 2 \\
\hline$B_{4}\left(4^{3} F_{4}\right)$ & $4^{+}$ & 7230 & 0 & 0 & 0 & 2 & 0 & 0 & 0 & 1 & 0 & 0 & 0 & 0 \\
\hline
\end{tabular}
$42 \mathrm{MeV}$. In this latter case, the $B_{1}\left(1 P_{1}\right)-B_{1}\left(1 P_{1}^{\prime}\right)$ mixing is not introduced because the masses of the two mesons differ by $60 \mathrm{MeV}$, approximately. 


$$
|n P\rangle=\cos \theta_{n P}\left|n^{1} P_{1}\right\rangle+\sin \theta_{n P}\left|n^{3} P_{1}\right\rangle
$$

and

$$
\left|n P^{\prime}\right\rangle=-\sin \theta_{n P}\left|n^{1} P_{1}\right\rangle+\cos \theta_{n P}\left|n^{3} P_{1}\right\rangle .
$$

For more details, see Refs. [50,51].

The spectrum of open-charm and open-bottom states, obtained by solving the eigenvalue problem of Eq. (4) with the values of the model paramaters of Ref. [50], is reported in Tables III-VIII, third columns.

\section{RESULTS AND DISCUSSION}

Below, we discuss our results of Tables III-VIII for the open-flavor strong decays of open-charm $\left(D\right.$ and $\left.D_{\mathrm{s}}\right)$ and open-bottom $\left(B, B_{\mathrm{s}}\right.$ and $\left.B_{\mathrm{c}}\right)$ mesons. The decays are computed in the ${ }^{3} P_{0}$ pair-creation model formalism of Sec. II A, with the values of the model parameters of Table II and Refs. [43-45]. There, the parameters of the $\mathrm{SU}_{\mathrm{f}}(4)$ and $\mathrm{SU}_{\mathrm{f}}(5)$ sectors were fitted to the charmonium and bottomonium strong decay amplitudes, respectively. When available, we calculate the amplitudes by using the experimental values of the meson masses, extracted from the particle data group (PDG) [10]; otherwise, we use the

TABLE VII. As Table III, but for $B_{s}$ mesons. The calculated mixing angles are $\theta_{1 P}=39.1^{\circ}, \theta_{2 P}=33.1^{\circ}, \theta_{3 P}=31.6^{\circ}, \theta_{1 D}=40.0^{\circ}$, $\theta_{2 D}=39.5^{\circ}$, and $\theta_{3 D}=39.1^{\circ}$. In the $B_{s 1}(5830)$ case, the value in brackets is calculated by using the experimental value for the mass,

\begin{tabular}{|c|c|c|c|c|c|c|c|c|c|c|}
\hline State & $J^{P}$ & Mass $(\mathrm{MeV})$ & $B K$ & $B^{*} K$ & $B K^{*}$ & $B^{*} K^{*}$ & $B_{s} \eta^{\prime}$ & $B_{s}^{*} \eta^{\prime}$ & $B_{s} \phi$ & $B_{s}^{*} \phi$ \\
\hline$B_{s 1}(5830)$ or $B_{s 1}\left(1 P_{1}\right)$ & $1^{+}$ & 5857,5829 & $\cdots$ & $85(30)$ & $\cdots$ & $\cdots$ & $\cdots$ & $\cdots$ & $\cdots$ & $\cdots$ \\
\hline$B_{s 0}\left(1^{3} P_{0}\right)$ & $0^{+}$ & 5830 & 208 & $\ldots$ & $\cdots$ & $\ldots$ & $\cdots$ & $\ldots$ & $\ldots$ & $\cdots$ \\
\hline$B_{s 2}^{*}(5840)$ or $B_{s 2}\left(1^{3} P_{2}\right)$ & $2^{+}$ & $5875,5840^{\dagger}$ & 1 & 0 & $\cdots$ & $\cdots$ & $\cdots$ & $\cdots$ & $\cdots$ & $\cdots$ \\
\hline$B_{s 1}\left(1 P_{1}^{\prime}\right)$ & $1^{+}$ & 5861 & $\cdots$ & 98 & $\cdots$ & $\cdots$ & $\cdots$ & $\cdots$ & $\cdots$ & $\cdots$ \\
\hline$B_{s 0}\left(2{ }^{1} S_{0}\right)$ & $0^{-}$ & 5985 & $\cdots$ & 106 & $\cdots$ & $\cdots$ & $\cdots$ & $\cdots$ & $\cdots$ & $\cdots$ \\
\hline$B_{s 1}\left(2^{3} S_{1}\right)$ & $1^{-}$ & 6013 & 46 & 81 & $\cdots$ & $\ldots$ & $\ldots$ & $\ldots$ & $\ldots$ & $\ldots$ \\
\hline$B_{s 2}\left(1 D_{2}\right)$ & $2^{-}$ & 6169 & $\cdots$ & 82 & 3 & $\cdots$ & $\cdots$ & $\cdots$ & $\cdots$ & $\cdots$ \\
\hline$B_{s 3}\left(1^{3} D_{3}\right)$ & $3^{-}$ & 6178 & 17 & 16 & 0 & $\cdots$ & $\cdots$ & $\cdots$ & $\cdots$ & $\cdots$ \\
\hline$B_{s 1}\left(1^{3} D_{1}\right)$ & $1^{-}$ & 6181 & 89 & 47 & 1 & $\cdots$ & $\cdots$ & $\cdots$ & $\cdots$ & $\cdots$ \\
\hline$B_{s 2}\left(1 D_{2}^{\prime}\right)$ & $2^{-}$ & 6196 & $\ldots$ & 86 & 3 & $\ldots$ & $\ldots$ & $\ldots$ & $\ldots$ & $\ldots$ \\
\hline$B_{s 0}\left(2^{3} P_{0}\right)$ & $0^{+}$ & 6279 & 52 & $\cdots$ & $\cdots$ & 71 & $\cdots$ & $\cdots$ & $\cdots$ & $\cdots$ \\
\hline$B_{s 1}\left(2 P_{1}\right)$ & $1^{+}$ & 6279 & $\cdots$ & 70 & 47 & 81 & $\cdots$ & $\ldots$ & $\ldots$ & $\ldots$ \\
\hline$B_{s 2}\left(2^{3} P_{2}\right)$ & $2^{+}$ & 6295 & 35 & 56 & 24 & 172 & $\ldots$ & $\ldots$ & $\cdots$ & $\cdots$ \\
\hline$B_{s 1}\left(2 P_{1}^{\prime}\right)$ & $1^{+}$ & 6296 & $\cdots$ & 63 & 49 & 51 & $\cdots$ & $\cdots$ & $\cdots$ & $\cdots$ \\
\hline$B_{s 0}\left(3^{1} S_{0}\right)$ & $0^{-}$ & 6409 & $\cdots$ & 16 & 24 & 92 & $\cdots$ & 6 & 4 & $\cdots$ \\
\hline$B_{s 1}\left(3^{3} S_{1}\right)$ & $1^{-}$ & 6429 & 10 & 21 & 10 & 84 & 5 & 6 & 6 & $\cdots$ \\
\hline$B_{s 4}\left(1^{3} F_{4}\right)$ & $4^{+}$ & 6431 & 28 & 27 & 4 & 125 & 0 & 0 & 0 & $\cdots$ \\
\hline$B_{s 2}\left(1^{3} F_{2}\right)$ & $2^{+}$ & 6453 & 46 & 34 & 39 & 38 & 3 & 1 & 1 & 0 \\
\hline$B_{s 2}\left(2 D_{2}\right)$ & $2^{-}$ & 6526 & $\cdots$ & 44 & 36 & 83 & $\cdots$ & 6 & 7 & 9 \\
\hline$B_{s 3}\left(2^{3} D_{3}\right)$ & $3^{-}$ & 6534 & 18 & 29 & 39 & 53 & 2 & 1 & 1 & 20 \\
\hline$B_{s 1}\left(2^{3} D_{1}\right)$ & $1^{-}$ & 6542 & 31 & 13 & 0 & 145 & 4 & 3 & 4 & 6 \\
\hline$B_{s 2}\left(2 D_{2}^{\prime}\right)$ & $2^{-}$ & 6553 & $\ldots$ & 43 & 33 & 87 & $\cdots$ & 7 & 7 & 8 \\
\hline$B_{s 1}\left(3 P_{1}\right)$ & $1^{+}$ & 6635 & $\cdots$ & 16 & 3 & 10 & & 5 & 5 & 12 \\
\hline$B_{s 0}\left(3^{3} P_{0}\right)$ & $0^{+}$ & 6638 & 13 & $\ldots$ & $\cdots$ & 10 & 5 & $\cdots$ & $\ldots$ & 21 \\
\hline$B_{s 2}\left(3^{3} P_{2}\right)$ & $2^{+}$ & 6647 & 12 & 14 & 0 & 19 & 2 & 4 & 4 & 11 \\
\hline$B_{s 1}\left(3 P_{1}^{\prime}\right)$ & $1^{+}$ & 6650 & $\ldots$ & 14 & 4 & 5 & $\ldots$ & 5 & 5 & 14 \\
\hline$B_{s 3}\left(1^{3} G_{3}\right)$ & $3^{-}$ & 6685 & 26 & 22 & 31 & 67 & 4 & 2 & 2 & 2 \\
\hline$B_{s 4}\left(2^{3} F_{4}\right)$ & $4^{+}$ & 6747 & 7 & 13 & 26 & 37 & 3 & 3 & 3 & 9 \\
\hline$B_{s 0}\left(4{ }^{1} S_{0}\right)$ & $0^{-}$ & 6757 & $\cdots$ & 2 & 6 & 22 & $\cdots$ & 2 & 2 & 2 \\
\hline$B_{s 2}\left(2^{3} F_{2}\right)$ & $2^{+}$ & 6768 & 21 & 13 & 4 & 110 & 0 & 1 & 1 & 11 \\
\hline$B_{s 2}\left(3 D_{2}\right)$ & $2^{-}$ & 6841 & $\cdots$ & 15 & 4 & 5 & & 2 & 2 & 5 \\
\hline$B_{s 3}\left(3^{3} D_{3}\right)$ & $3^{-}$ & 6848 & 10 & 12 & 4 & 7 & 0 & 1 & 1 & 6 \\
\hline$B_{s 2}\left(3 D_{2}^{\prime}\right)$ & $2^{-}$ & 6864 & $\ldots$ & 14 & 3 & 5 & $\ldots$ & 2 & 2 & 5 \\
\hline$B_{s 0}\left(4^{3} P_{0}\right)$ & $0^{+}$ & 6949 & 2 & $\ldots$ & . & 10 & 1 & $\ldots$ & .. & 4 \\
\hline$B_{s 3}\left(2^{3} G_{3}\right)$ & $3^{-}$ & 6970 & 15 & 10 & 6 & 62 & 0 & 0 & 0 & 10 \\
\hline$B_{s 4}\left(3^{3} F_{4}\right)$ & $4^{+}$ & 7034 & 8 & 9 & 5 & 5 & 0 & 0 & 0 & 3 \\
\hline$B_{s 4}\left(4^{3} F_{4}\right)$ & $4^{+}$ & 7297 & 3 & 2 & 0 & 3 & 0 & 0 & 0 & 1 \\
\hline
\end{tabular}
without mixing with $B_{s 1}\left(1 P_{1}^{\prime}\right)$. 
relativized QM predictions reported in the third columns of Tables III and IV, and VI-VIII. See also Table I, which shows the existing experimental data for the total widths of $D, D_{\mathrm{s}}, B$, and $B_{\mathrm{s}}$ resonances. There are no data available for higher $B_{\mathrm{c}}$ resonances [10]. Our theoretical results reproduce the global trend of the PDG data [10] with a few exceptions.

In more detail, starting from the $D$ sector, our result for the open-flavor width of the $D^{*}(2007)^{0}, \Gamma_{\text {of }}^{\text {th }}=4 \mathrm{keV}$, is compatible with the total experimental width $\Gamma_{\text {tot }}^{\text {exp }}<$ $2.1 \mathrm{MeV}$ [10]. A more refined prediction would require the introduction of coupled-channel effects, the mass of $D^{*}(2007)^{0}$ being very close to the $D \pi$ threshold. The same applies to $D_{0}^{*}(2400)$, where the presence of higher Fock components in the meson wave function may lower the relativized QM prediction for the mass, $2398 \mathrm{MeV}$, down to the experimental value, $2318 \pm 29 \mathrm{MeV}$, and also contribute to the open-flavor amplitude. In the $D_{1}(2420)$ case, which should mainly decay into $D^{*} \pi$ with the possible chain $D^{*} \pi \rightarrow D \pi \pi$, our ${ }^{3} P_{0}$ model prediction is compatible with the data, while this is not true for $D_{1}(2430)$, being $\Gamma_{\mathrm{of}}^{\mathrm{th}} \ll \Gamma_{\text {tot }}^{\exp }$. Nevertheless, it is worth noting that, in this second case, the experimental error is still very large; moreover, if $D_{1}(2420)$ and $D_{1}(2430)$ are mixed by spinorbit forces, their open-flavor widths are likely to be of the same order of magnitude. Our results for the total openflavor widths of $D_{2}^{*}(2460)$ and $D_{0}(2550)$ are compatible with the present experimental data, being $\Gamma_{\text {of }}^{\text {th }}<\Gamma_{\text {tot }}^{\exp }$. In the $D_{2}^{*}(2460)$ case, it is also interesting to calculate the ratio between the kinematically allowed open-flavor decays, namely $\mathcal{R}_{D_{2}^{*}(2460)}^{\text {of }}=\Gamma_{D_{2}^{*}(2460) \rightarrow D \pi} / \Gamma_{D_{2}^{*}(2460) \rightarrow D^{*} \pi}$. Our estimate, $\mathcal{R}_{D_{2}^{*}(2460)}^{\text {of }} \approx 3$, is close to the heavy meson effective theory one of Ref. [27], i.e., 2.29. Our result can also be compared to the existing experimental data: $3.0 \pm$ $1.1 \pm 1.5[61], 2.2 \pm 0.7 \pm 0.6[62], 2.8 \pm 0.8_{-0.6}^{+0.5}$ [63], and $1.47 \pm 0.03 \pm 0.16$ [16]. Other interesting information is the ratio between the total decay widths of $D(2550)$ and $D(2600)$, under the hypothesis that $D(2600)$ has $2^{3} S_{1}$ quantum numbers. Also, in this second case, our result, $\mathcal{R}_{D(2550) / D(2600)}^{\text {of }}=\Gamma_{D(2550)}^{\text {of }} / \Gamma_{D(2600)}^{\text {of }}=0.64$, is similar to the heavy meson effective theory one of Ref. [23], $\mathcal{R}_{D(2550) / D(2600)}^{\text {of }}=0.85$.

Moving to the $D_{\mathrm{s}}$ sector, our predictions for $D_{\mathrm{s} 1}(2460)$ are compatible with the data [10], while those for $D_{\mathrm{s} 1}(2536)$ are not. The former meson has a narrow width and mainly decays to $D_{\mathrm{s}}^{*}$ via photon or $\pi^{0}$ emission, which are normally suppressed decay modes [6]. Because of the large mass difference between $D_{\mathrm{s} 1}(2460)$ and $D_{\mathrm{s} 1}(2536)$, which cannot be explained in terms of hyperfine or spin-orbit splittings, these mesons may have exotic nature. Our results for the total widths of $D_{\mathrm{s} 2}^{*}(2573), D_{\mathrm{s} 1}^{*}(2700), D_{\mathrm{s} 1}^{*}(2860)$, and $D_{\mathrm{s} 3}^{*}(2860)$ are compatible with the experimental data [10], being $\Gamma_{\text {of }}^{\text {th }}<\Gamma_{\text {tot }}^{\text {exp }}$. We cannot say much on the single channels, as the PDG only provides some preliminary results for a few branching fractions, except that, in the $D_{\mathrm{s} 2}^{*}(2573)$ case, our predictions are compatible with $\Gamma\left(D^{*} K\right) / \Gamma(D K)<0.33$ [10]. In the $D_{\mathrm{s} 3}^{*}(2860)$ case, we also show predictions extracted by using the relativized QM mass for the decaying meson because I) There is a large difference between experimental and calculated masses and II) the experimental data are not very reliable as, at the moment, the state is excluded from the PDG summary table [10].

Finally, we discuss our predictions for the $B$ and $B_{\mathrm{s}}$ sectors. Our results for the total open-flavor widths of

TABLE VIII. As Table III, but for $B_{c}$ mesons.

\begin{tabular}{|c|c|c|c|c|c|c|c|c|c|c|}
\hline State & $J^{P}$ & Mass $(\mathrm{MeV})$ & $B D$ & $B^{*} D$ & $B D^{*}$ & $B^{*} D^{*}$ & $B_{s} D_{s}$ & $B_{s}^{*} D_{s}$ & $B_{s} D_{s}^{*}$ & $B_{s}^{*} D_{s}^{*}$ \\
\hline$B_{c 2}\left(2^{3} P_{2}\right)$ & $2^{+}$ & 7164 & 2 & $\cdots$ & $\cdots$ & $\ldots$ & $\ldots$ & $\cdots$ & $\cdots$ & $\ldots$ \\
\hline$B_{c 0}\left(3{ }^{1} S_{0}\right)$ & $0^{-}$ & 7249 & $\ldots$ & 107 & $\ldots$ & $\cdots$ & $\cdots$ & $\cdots$ & $\cdots$ & $\cdots$ \\
\hline$B_{c 2}\left(1^{3} F_{2}\right)$ & $2^{+}$ & 7269 & 90 & 29 & $\cdots$ & $\cdots$ & $\cdots$ & $\cdots$ & $\cdots$ & $\cdots$ \\
\hline$B_{c 4}\left(1^{3} F_{4}\right)$ & $4^{+}$ & 7271 & 3 & 1 & $\ldots$ & $\cdots$ & $\ldots$ & $\cdots$ & $\cdots$ & $\cdots$ \\
\hline$B_{c 1}\left(3^{3} S_{1}\right)$ & $1^{-}$ & 7272 & 13 & 64 & $\ldots$ & $\cdots$ & $\ldots$ & $\cdots$ & $\cdots$ & $\cdots$ \\
\hline$B_{c 1}\left(2^{3} D_{1}\right)$ & $1^{-}$ & 7365 & 3 & 2 & 37 & 27 & 10 & $\cdots$ & $\cdots$ & $\cdots$ \\
\hline$B_{c 3}\left(2^{3} D_{3}\right)$ & $3^{-}$ & 7379 & 46 & 52 & 15 & 184 & 0 & $\ldots$ & $\cdots$ & $\cdots$ \\
\hline$B_{c 0}\left(3^{3} P_{0}\right)$ & $0^{+}$ & 7454 & 0 & - & - & 157 & 4 & $\ldots$ & $\cdots$ & $\ldots$ \\
\hline$B_{c 3}\left(1^{3} G_{3}\right)$ & $3^{-}$ & 7474 & 93 & 66 & 45 & 27 & 5 & 1 & $\cdots$ & $\cdots$ \\
\hline$B_{c 2}\left(3^{3} P_{2}\right)$ & $2^{+}$ & 7487 & 10 & 4 & 7 & 105 & 4 & 7 & 0 & $\cdots$ \\
\hline$B_{c 2}\left(2^{3} F_{2}\right)$ & $2^{+}$ & 7565 & 36 & 13 & 0 & 133 & 1 & 3 & 4 & 1 \\
\hline$B_{c 0}\left(4^{1} S_{0}\right)$ & $0^{-}$ & 7567 & $\cdots$ & 2 & 23 & 55 & $\ldots$ & 2 & 2 & 14 \\
\hline$B_{c 4}\left(2^{3} F_{4}\right)$ & $4^{+}$ & 7568 & 21 & 34 & 39 & 52 & 4 & 3 & 0 & 6 \\
\hline$B_{c 3}\left(3^{3} D_{3}\right)$ & $3^{-}$ & 7669 & 20 & 20 & 6 & 19 & 0 & 1 & 3 & 5 \\
\hline$B_{c 0}\left(4^{3} P_{0}\right)$ & $0^{+}$ & 7740 & 4 & $\cdots$ & $\cdots$ & 22 & 3 & $\cdots$ & . & 1 \\
\hline$B_{c 3}\left(2^{3} G_{3}\right)$ & $3^{-}$ & 7743 & 42 & 25 & 12 & 104 & 0 & 0 & 1 & 9 \\
\hline$B_{c 4}\left(3^{3} F_{4}\right)$ & $4^{+}$ & 7834 & 15 & 19 & 15 & 12 & 1 & 0 & 0 & 6 \\
\hline$\underline{B_{c 4}\left(4^{3} F_{4}\right)}$ & $4^{+}$ & 8077 & 10 & 12 & 7 & 6 & 0 & 0 & 0 & 3 \\
\hline
\end{tabular}


$B_{2}^{*}(5747)$ and $B_{s 2}^{*}(5840)$ and for the ratio $\frac{\Gamma\left(B_{2}^{*}(5747) \rightarrow B^{*} \pi\right)}{\Gamma\left(B_{2}^{*}(5747) \rightarrow B \pi\right)}$ are compatible with the experimental data $[10,64]$. By contrast, our result for the open-flavor width of $B_{s 1}(5830)$ is incompatible with the data. Our prediction is very sensitive to the value of the decaying meson mass - as $B_{s 1}(5830)$ is close to the $B^{*} K$ threshold - and thus a few $\mathrm{MeV}$ mass difference can produce large deviations in the calculated decay amplitude. It is also interesting to discuss the possible quantum number assignments for $B_{J}(5970)^{0}$, the width of which is $81 \pm 12 \mathrm{MeV}$ [10]. Following Ref. [26], the three possible assignments are $2^{3} S_{1}, 1^{3} D_{3}$, and $1^{3} D_{1}$. According to our results, the most likely is $2^{3} S_{1}$.

\section{SUMMARY AND CONCLUSION}

We computed the open-flavor strong decays of opencharm and open-bottom mesons within a modified version of the ${ }^{3} P_{0}$ pair-creation model $[29,38]$.

In the ${ }^{3} P_{0}$ model, the open-flavor decays take place in the rest frame of the initial state, via the production of a light $q \bar{q}$ pair (i.e., $q=u, d$, or $s$ ) with ${ }^{3} P_{0}$ quantum numbers. Heavy quark pair production is heavily suppressed, as required by the phenomenology, by substituting the pair-creation strength, $\gamma_{0}$, with an effective one, $\gamma_{0}^{\text {eff }}[34,43-45,52,60]$. Moreover, the nonpointlike nature of the pair of produced quarks is taken into account by introducing a quark form factor $[43-45,52,60,66-70]$ into the model transition operator. The values of the ${ }^{3} P_{0}$ model parameters in the $\mathrm{SU}_{\mathrm{f}}(4)$ and $\mathrm{SU}_{\mathrm{f}}(5)$ sectors were extracted from our previous studies on $c \bar{c}[43,44]$ and $b \bar{b}[44,45]$ meson spectroscopy and decays, where they were fitted to the existing experimental data [10].

The open-charm and open-bottom meson spectra we needed in our calculation were predicted within Godfrey and Isgur's relativized quark model [50]. This is one of the most powerful tools for the study of $q \bar{q}$ meson spectroscopy and provides a description of the meson spectrum in the light, strange, $c \bar{c}$, etc., sectors with a universal set of parameters; moreover, 30 years since its formulation, it still gives a good overall description of the experimental data.

As discussed in our previous papers [43-45,58], there may be substantial deviations between the experimental values of the masses and QM predictions [50] in the case of resonances lying close to meson-meson decay thresholds. In these cases, continuum-coupling effects may be important and determine a downward energy shift for the bare meson masses, thus improving the fit to the data; coupledchannel effects may also contribute to the open-flavor amplitudes. Such resonances may have an exotic nature, such as tetraquarks, meson-meson molecules, or $q \bar{q}$ mesons plus continuum components. For example, this may be the case of $D\left(1^{3} S_{1}\right), D_{0}^{*}(2400)$, and $D_{s 0}^{*}(2317)$ [53], where $\mathrm{QM}$ predictions are incompatible with the present experimental data [10]. The possible interpretations for suspected exotic open-charm and open-bottom mesons will be discussed in a future paper.
In conclusion, we think that our predictions can be useful to the experimentalists in their study of the properties of open-charm and open-bottom mesons and in the search for new resonances.

\section{APPENDIX: FLAVOR COUPLINGS IN THE ${ }^{3} \boldsymbol{P}_{0}$ PAIR-CREATION MODEL}

In the following, we show how to calculate the $\mathrm{SU}_{\mathrm{f}}(5)$ flavor couplings of the ${ }^{3} P_{0}$ pair-creation model. The $\mathrm{SU}_{\mathrm{f}}(4)$ couplings can be computed analogously.

We consider the transition $A \rightarrow B C$, where $A, B$, and $C$ are quark-antiquark mesons. The $\mathrm{SU}_{\mathrm{f}}(5)$ flavor couplings can be written as the scalar product between initial, $\left|A\left(q_{1} \bar{q}_{2}\right) \Phi_{0}\left(q_{3} \bar{q}_{4}\right)\right\rangle$, and final, $\left|B\left(q_{1} \bar{q}_{4}\right) C\left(q_{3} \bar{q}_{2}\right)\right\rangle$, states. Here, $\Phi_{0}$ is the $\operatorname{SU}_{\mathrm{f}}(5)$ flavor singlet,

$$
\begin{aligned}
\left|\Phi_{0}\right\rangle & =\frac{1}{\sqrt{n_{\mathrm{f}}}} \sum_{i=1}^{n_{\mathrm{f}}} q_{3}^{i} \bar{q}_{4}^{i} \\
& =\frac{1}{\sqrt{5}}(|u \bar{u}\rangle+|d \bar{d}\rangle+|s \bar{s}\rangle+|c \bar{c}\rangle+|b \bar{b}\rangle),
\end{aligned}
$$

and $n_{\mathrm{f}}=5$ is the dimension of the $\mathrm{SU}_{\mathrm{f}}$ flavor group. In general, two different diagrams can contribute to the flavor matrix element $\left\langle B C \mid A \Phi_{0}\right\rangle$ : in the first one, the quark in $A$ ends up in $B$, while in the second one, it ends up in $C$. In the majority of cases, one of these two diagrams vanishes; however, for some matrix elements, both must be taken into account $[43-45,52]$. Finally, the flavor matrix elements can be calculated as

$$
\begin{aligned}
& {\left[\left\langleq_{1} \bar{q}_{4}\left|\otimes\left\langle q_{3} \bar{q}_{2}\right|\right]\left[\left|q_{1} \bar{q}_{2}\right\rangle \otimes \frac{1}{\sqrt{n_{\mathrm{f}}}} \sum_{i=1}^{n_{\mathrm{f}}}\left|q_{3}^{i} \bar{q}_{4}^{i}\right\rangle\right]\right.\right.} \\
& =\frac{1}{\sqrt{n_{\mathrm{f}}}} \sum_{i=1}^{n_{\mathrm{f}}}\left[\left\langle q_{1} \bar{q}_{4} q_{3} \bar{q}_{2} \mid q_{1} \bar{q}_{2} q_{3}^{i} \bar{q}_{4}^{i}\right\rangle\right. \\
& \left.\quad+\left\langle q_{3} \bar{q}_{2} q_{1} \bar{q}_{4} \mid q_{1} \bar{q}_{2} q_{3}^{i} \bar{q}_{4}^{i}\right\rangle\right] .
\end{aligned}
$$

For an example, we calculate the $B^{0} \rightarrow B^{0} \pi^{0}$ flavor coupling. The flavor matrix element can be written as

$$
\begin{aligned}
\left\langle B^{0} \pi^{0} \mid B^{0} \Phi_{0}\right\rangle_{\text {flavor }}= & -\frac{1}{\sqrt{10}}[\langle d \bar{b} d \bar{d} \mid d \bar{b} d \bar{d}\rangle+\langle d \bar{d} d \bar{b} \mid d \bar{b} d \bar{d}\rangle \\
& -\langle d \bar{b} u \bar{u} \mid d \bar{b} u \bar{u}\rangle+\cdots] \\
= & -\frac{1}{\sqrt{10}} .
\end{aligned}
$$

The only surviving contribution in Eq. (A3) is $\langle d \bar{d} d \bar{b} \mid d \bar{b} d \bar{d}\rangle$; the others, like $\langle d \bar{b} d \bar{d} \mid d \bar{b} d \bar{d}\rangle$ or $\langle d \bar{b} u \bar{u} \mid d \bar{b} u \bar{u}\rangle$, are null [see Eq. (A2)]. In conclusion, after dividing Eq. (A3) by the corresponding SU(2) (isospin) Clebsch-Gordan coefficient, we get

$$
\left\langle B \pi \mid B \Phi_{0}\right\rangle_{\text {flavor }}=-\sqrt{\frac{3}{10}} .
$$


[1] S. K. Choi et al. (Belle Collaboration), Phys. Rev. Lett. 91, 262001 (2003).

[2] R. Aaij et al. (LHCb Collaboration), Phys. Rev. Lett. 110, 222001 (2013).

[3] G. Aad et al. (ATLAS Collaboration), Phys. Rev. Lett. 108, 152001 (2012).

[4] V. M. Abazov et al. (D0 Collaboration), Phys. Rev. D 86, 031103 (2012).

[5] B. Aubert et al. (BABAR Collaboration), Phys. Rev. Lett. 90, 242001 (2003).

[6] D. Besson et al. (CLEO Collaboration), Phys. Rev. D 68, 032002 (2003); 75, 119908 (2007).

[7] C. Bebek et al. (CLEO Collaboration), Phys. Rev. Lett. 46, 84 (1981).

[8] J. Lee-Franzini, U. Heintz, D. M. J. Lovelock, M. Narain, R. D. Schamberger, J. Willins, C. Yanagisawa, P. Franzini, and P. M. Tuts, Phys. Rev. Lett. 65, 2947 (1990).

[9] F. Abe et al. (CDF Collaboration), Phys. Rev. D 58, 112004 (1998).

[10] C. Patrignani et al. (Particle Data Group Collaboration), Chin. Phys. C 40, 100001 (2016).

[11] N. Brambilla et al., Eur. Phys. J. C 71, 1534 (2011).

[12] J. M. Link et al. (FOCUS Collaboration), Phys. Lett. B 586, 11 (2004).

[13] K. Abe et al. (Belle Collaboration), Phys. Rev. D 69, 112002 (2004).

[14] V. M. Abazov et al. (D0 Collaboration), Phys. Rev. Lett. 99, 172001 (2007).

[15] T. Aaltonen et al. (CDF Collaboration), Phys. Rev. Lett. 102, 102003 (2009).

[16] P. del Amo Sanchez et al. (BABAR Collaboration), Phys. Rev. D 82, 111101 (2010).

[17] R. Aaij et al. (LHCb Collaboration), J. High Energy Phys. 09 (2013) 145.

[18] T. A. Aaltonen et al. (CDF Collaboration), Phys. Rev. D 90, 012013 (2014).

[19] U. Kilian, J. G. Korner, and D. Pirjol, Phys. Lett. B 288, 360 (1992).

[20] P. Colangelo, F. De Fazio, G. Nardulli, N. Di Bartolomeo, and R. Gatto, Phys. Rev. D 52, 6422 (1995).

[21] R. Casalbuoni, A. Deandrea, N. Di Bartolomeo, R. Gatto, F. Feruglio, and G. Nardulli, Phys. Rep. 281, 145 (1997).

[22] P. Colangelo, F. De Fazio, S. Nicotri, and M. Rizzi, Phys. Rev. D 77, 014012 (2008).

[23] Z. G. Wang, Phys. Rev. D 83, 014009 (2011).

[24] P. Colangelo, F. De Fazio, F. Giannuzzi, and S. Nicotri, Phys. Rev. D 86, 054024 (2012).

[25] Z. G. Wang, Phys. Rev. D 88, 114003 (2013).

[26] Z. G. Wang, Eur. Phys. J. Plus 129, 186 (2014).

[27] Z. G. Wang, Commun. Theor. Phys. 66, 671 (2016).

[28] S. Capstick and W. Roberts, Prog. Part. Nucl. Phys. 45, S241 (2000).

[29] L. Micu, Nucl. Phys. B10, 521 (1969).

[30] R. Kokoski and N. Isgur, Phys. Rev. D 35, 907 (1987).

[31] S. Kumano and V. R. Pandharipande, Phys. Rev. D 38, 146 (1988).

[32] F. Stancu and P. Stassart, Phys. Rev. D 38, 233 (1988); 39, 343 (1989); 41, 916 (1990); 42, 1521 (1990).

[33] P. Geiger and E. S. Swanson, Phys. Rev. D 50, 6855 (1994).

[34] Y.S. Kalashnikova, Phys. Rev. D 72, 034010 (2005).
[35] J. Segovia, D. R. Entem, and F. Fernandez, Phys. Lett. B 715, 322 (2012).

[36] H. G. Blundell and S. Godfrey, Phys. Rev. D 53, 3700 (1996).

[37] E. S. Ackleh, T. Barnes, and E. S. Swanson, Phys. Rev. D 54, 6811 (1996); T. Barnes, F. E. Close, P. R. Page, and E. S. Swanson, Phys. Rev. D 55, 4157 (1997).

[38] A. Le Yaouanc, L. Oliver, O. Pene, and J.-C. Raynal, Phys. Rev. D 8, 2223 (1973); 9, 1415 (1974).

[39] S. Capstick and W. Roberts, Phys. Rev. D 47, 1994 (1993); 49, 4570 (1994); 58, 074011 (1998).

[40] R. Bijker, J. Ferretti, G. Galatà, H. García-Tecocoatzi, and E. Santopinto, Phys. Rev. D 94, 074040 (2016); H. García-Tecocoatzi, R. Bijker, J. Ferretti, G. Galatà, and E. Santopinto, Few-Body Syst. 57, 985 (2016).

[41] A. Le Yaouanc, L. Oliver, O. Pene, and J.-C. Raynal, Phys. Lett. B 71, 397 (1977); 72, 57 (1977).

[42] T. Barnes, S. Godfrey, and E. S. Swanson, Phys. Rev. D 72, 054026 (2005).

[43] J. Ferretti, G. Galatà, and E. Santopinto, Phys. Rev. C 88, 015207 (2013).

[44] J. Ferretti, G. Galatà, and E. Santopinto, Phys. Rev. D 90, 054010 (2014).

[45] J. Ferretti and E. Santopinto, Phys. Rev. D 90, 094022 (2014).

[46] S. Godfrey and K. Moats, Phys. Rev. D 92, 054034 (2015).

[47] F. E. Close and E. S. Swanson, Phys. Rev. D 72, 094004 (2005).

[48] S. Godfrey and K. Moats, Phys. Rev. D 93, 034035 (2016).

[49] S. Godfrey, K. Moats, and E. S. Swanson, Phys. Rev. D 94, 054025 (2016).

[50] S. Godfrey and N. Isgur, Phys. Rev. D 32, 189 (1985).

[51] S. Godfrey, Phys. Rev. D 70, 054017 (2004).

[52] J. Ferretti, G. Galatá, E. Santopinto, and A. Vassallo, Phys. Rev. C 86, 015204 (2012).

[53] D. S. Hwang and D. W. Kim, Phys. Lett. B 601, 137 (2004).

[54] F. K. Guo and U. G. Meißner, Phys. Rev. Lett. 108, 112002 (2012).

[55] M. Cardoso, G. Rupp, and E. van Beveren, Eur. Phys. J. C 75, 26 (2015).

[56] F. K. Guo, U. G. Meißner, and Z. Yang, Phys. Lett. B 760, 417 (2016).

[57] Y. Lu, M. N. Anwar, and B. S. Zou, Phys. Rev. D 94, 034021 (2016).

[58] J. Ferretti and E. Santopinto, arXiv:1806.02489.

[59] W. Roberts and B. Silvestre-Brac, Few-Body Syst. 11, 171 (1992).

[60] H. García-Tecocoatzi, R. Bijker, J. Ferretti, and E. Santopinto, Eur. J. Phys. 53, 115 (2017).

[61] H. Albrecht et al. (ARGUS Collaboration), Phys. Lett. B 232, 398 (1989).

[62] P. Avery et al. (CLEO Collaboration), Phys. Lett. B 331, 236 (1994).

[63] S. Chekanov et al. (ZEUS Collaboration), Eur. Phys. J. C 60, 25 (2009).

[64] R. Aaij et al. (LHCb Collaboration), J. High Energy Phys. 04 (2015) 024. 
[65] B. Aubert et al. (BABAR Collaboration), Phys. Rev. D 80, 092003 (2009).

[66] P. Geiger and N. Isgur, Phys. Rev. Lett. 67, 1066 (1991); Phys. Rev. D 44, 799 (1991); 47, 5050 (1993).

[67] P. Geiger and N. Isgur, Phys. Rev. D 55, 299 (1997).
[68] R. Bijker and E. Santopinto, Phys. Rev. C 80, 065210 (2009).

[69] E. Santopinto and R. Bijker, Phys. Rev. C 82, 062202 (2010).

[70] R. Bijker, J. Ferretti, and E. Santopinto, Phys. Rev. C 85, 035204 (2012). 